\title{
Estimation of ochratoxin A in portuguese population: New data on the occurrence in human urine by high performance liquid chromatography with fluorescence detection
}

\author{
A. Pena ${ }^{\mathrm{a}, *}$, M. Seifrtová ${ }^{\mathrm{a}}$, C. Lino ${ }^{\mathrm{a}}$, I. Silveira ${ }^{\mathrm{a}}$, P. Solich ${ }^{\mathrm{b}}$ \\ ${ }^{a}$ Group of Bromatology, Centre of Pharmaceutical Studies, Faculty of Pharmacy, University of Coimbra, Rua do Norte, 3000 Coimbra, Portugal \\ ${ }^{\mathrm{b}}$ Department of Analytical Chemistry, Faculty of Pharmacy, Charles University, 50005 Hradec Králové, Czech Republic
}

Received 8 July 2005; accepted 28 April 2006

\begin{abstract}
With increasing knowledge of the persistence of OTA in the food chain, exposure to this mycotoxin is a potential human health hazard to humans, and evaluating its presence in populations has become highly important.

A sensitive and accurate analytical method for the determination of ochratoxin A in urine was validated, since is less invasive than blood monitoring. It involves extraction with $5 \% \mathrm{NaHCO}_{3}$, immunoaffinity column (IAC) for clean-up and high performance liquid chromatography with fluorescence detection (HPLC-FD). The limit of quantification was $0.02 \mathrm{ng} / \mathrm{mL} \mathrm{of} \mathrm{urine}(1.3 \mathrm{ng} / \mathrm{mL} \mathrm{of} \mathrm{the} \mathrm{extract}$ injected) and recovery of ochratoxin A from urine samples spiked at the three fortification levels, were higher than $90 \%$ with RSD lower than $9 \%$. The identification of OTA was confirmed by methyl ester derivatization and then HPLC analysis.

Based in ours first results we can assume that OTA conjugation with glucuronic acid in human urine occurs. In the present study, we follow up OTA levels in 60 urine samples of inhabitants from Coimbra city, Portugal, in order to evaluate population contamination, and the presence of OTA was found in 42 samples, at concentrations above the LOQ, ranged between 0.021 and $0.105 \mathrm{ng} / \mathrm{mL}$.

(C) 2006 Elsevier Ltd. All rights reserved.
\end{abstract}

Keywords: Food safety; Mycotoxins; Ochratoxin A; Urine; HPLC; Fluorescence detection

\section{Introduction}

Ochratoxin A (OTA) is a mycotoxin produced by Penicillium verrucosum, Aspergillus ochraceus and other related species (Álvarez et al., 2004). As to food safety, the problem of OTA lies in the fact that its presence in human body is due to the ingestion of small quantities present in several commodities, such as, coffee, cereals, rice, dried fruits, beer

Abbreviations: AOAC; BEN, Balkan endemic nephropathy; FD, fluorescence detection; HPLC, high performance liquid chromatography; IAC, immunoaffinity column; IARC, International Agency for Research on Cancer; MS, mass spectrometry; LOQ, limit of quantification; OTA, ochratoxin A; RSD, relative standard deviation; UTT, urinary tract tumors.

${ }^{*}$ Corresponding author. Tel.: +351 239 859994; fax: +351 239827126.

E-mail address: apena@ci.uc.pt (A. Pena). and wine (Visconti et al., 2000; Blesa et al., 2004; Monaci et al., 2004; Pena et al., 2005).

Toxicological profile includes nephrotoxicity, causing both acute and chronic lesions of kidneys and has been suspected to be involved in the aetiology of Balkan endemic nephropathy (BEN), a disease characterized by progressive renal fibrosis in humans mainly occurring in some areas of South-Eastern Europe, and its implication in the urinary tract tumors (UTT) (Pfohl-Leszkowicz et al., 2002; Gekle et al., 2005). OTA is classified as a possible carcinogen to human (Group 2B) by the International Agency for Research on Cancer (IARC, 1993).

When ingested as a food contaminant, OTA is frequently found in human blood due to the long elimination half-life (about 35 days in serum), as consequence of its binding to plasma proteins, its enterohepatic circulation 
and its re-absorption from urine (Studer-Rohr et al., 2000). This renders OTA among the most frequent mycotoxin contaminants in human blood in the world.

Human exposure to ochratoxin A has been clearly demonstrated by its detection in human blood, urine and breast milk (Zimmerli and Dick, 1995; Thuvander et al., 2001; Dinis et al., 2003a,b). In Portugal, higher OTA levels were observed in the rural populations compared with the urban populations of the zones studied (Dinis et al., 2003a), as well as the nephropathy individuals when compared with the healthy individuals (Dinis et al., 2003b).

Elimination in urine has been considered the main route of OTA excretion from the human body (Li et al., 1997). Urinary monitoring is relatively unexplored due to the lower concentrations involved. Recent developments in analytical methodology mean that urinary monitoring is now more feasible and it has the advantage of being less invasive than blood monitoring. However, data in the frequency and concentration of OTA in human urine are scarce.

The expected levels in human urine are at trace levels, and therefore sensitive and accurate methods are required. Liquid chromatography with fluorescence or mass spectrometry detection, coupled with immunoaffinity column (IAC) clean-up, is the most widely employed analytical methodology (Valenta, 1998; Blesa et al., 2004; Biffi et al., 2004; Pena et al., 2005). Owing to its good native fluorescence properties, OTA can be determined very sensitively with fluorescence detection.

In the present work, the validation of a simple, sensitive and accurate method for the urinary monitoring OTA levels as a marker for OTA human exposure is presented. Urine samples were extracted with $5 \%$ sodium hydrogen carbonate followed with clean-up through immunoaffinity column (IAC) and high performance liquid chromatography with fluorescence detection (HPLC-FD). Positive samples were confirmed by methylation of OTA.

As glucuronide conjugates with other mycotoxins, were found in urine, incubation with $\beta$-glucuronidase prior to the extraction was studied in naturally contaminated samples, and based in ours first results we can assume that OTA conjugation with glucuronic acid in human urine occurs.

The aim of our research was to add new data to the previous studies conducted in Portuguese population blood. Therefore, we follow up OTA levels in 60 urine samples of healthy individuals from Coimbra city, Portugal, for a better evaluation of Portuguese population contamination.

\section{Experimental}

\subsection{Solvents and materials}

HPLC grade acetonitrile and methanol were purchased from Carlo Erba (Milan, Italy). Acetic acid, $\beta$-glucoronidase, sodium hydrogen carbonate and sodium sulphate anhydrous obtained from Merck (Darmstadt, Germany).
Ochratoxin A and boron trifluoride 14\% methanolic solution were purchased from Sigma Chemicals Co. (St. Louis, USA).

Standard solution of ochratoxin A was prepared from the ochratoxin A vial. The standard stock solution $(250 \mu \mathrm{g} / \mathrm{mL})$ was made in $4 \mathrm{~mL}$ toluene-acetic acid (99:1), and stored at $-20^{\circ} \mathrm{C}$. Intermediate standard solution $(10 \mu \mathrm{g} / \mathrm{mL})$ was prepared diluting $1 \mathrm{~mL}$ of stock standard solution with $25 \mathrm{~mL}$ toluene-acetic acid (99:1). The actual concentration of the OTA was calculated using an ultraviolet light spectrophotometer set at $333 \mathrm{~nm}(\varepsilon=544)$ (Entwisle et al., 2000).

For fortification assays, standard solution was prepared in toluene:acetic acid (99:1) at $1000 \mathrm{ng} / \mathrm{mL}$. For calibration curve, the standard solutions were prepared evaporating $100 \mu \mathrm{L}$ of intermediate standard solution at dryness, and diluting to $10 \mathrm{~mL}$ with mobile phase $(100 \mathrm{ng} / \mathrm{mL})$. After suitable dilutions in water-methanol-acetic acid (49.5:49.5:1), the working standard solution was used to prepare the external calibration curve $(1,5,10,15$ and $20 \mathrm{ng} / \mathrm{mL}$ ).

Imunoaffinity columns Ochratest ${ }^{\mathrm{TM}}$ (Viacom/Watertown, USA). Centrifuge Meditronic S-599 (Selecta, Barcelona, Spain). Vortex mixer Retsh (Haan, Germany). Ultrasonic bath Sonorex RK 100 (Berlin, Germany).

Water was purified by distillation and passage through Milli Q system (Millipore, Bedford, MA). All chromatographic solvents and water were filtered through a $0.50 \mu \mathrm{m}$ filter under vacuum and degassed for $15 \mathrm{~min}$ in ultrasonic bath.

Amber glassware was used to prevent light. The decontamination of the glassware was made by a sodium hypochlorite solution. Then, it was acid washed by immersion of the glassware in a solution of $4 \mathrm{~mL} / \mathrm{L} \mathrm{H}_{2} \mathrm{SO}_{4}$ followed by washing to neutral $\mathrm{pH}$ by rinsing with distilled water.

\subsection{HPLC system and chromatographic conditions}

The HPLC apparatus consisted of a pump model 307 Gilson (Gilson Medical Electronics, Villiers-le-Bel, France), an injector Rheodyne model 7125 (Cotati, California, USA), and a Perkin Elmer spectrofluorimeter, model LS45 (Perkin Elmer, Beaconsfield, UK) operated at an excitation wavelength of $333 \mathrm{~nm}$ and an emission wavelength of $477 \mathrm{~nm}$. The spectral bandwidth was $10 \mathrm{~nm}$ for both excitation and emission. The results were recorded on a 3390A integrator (Hewlett Packard, Philadelphia, USA).

Column oven for control HPLC column temperature $\left(30 \pm 0.5^{\circ} \mathrm{C}\right)$. The results were recorded on a $3390 \mathrm{~A}$ integrator (Hewllet-Packard, Philadelphia, PA). A guard column, $\mathrm{C}_{18}-5 \mu \mathrm{m}$, Nucleosil $120 \mathrm{KS}(30 \times 4 \mathrm{~mm}$ i.d. $)$, and a column $\mathrm{C}_{18^{-}}-5 \mu \mathrm{m}$, Nucleosil $100 \mathrm{KS}(250 \times 4.6 \mathrm{~mm}$ i.d.) were used.

The mobile phase (acetronitrile/water/acetic acid 49.5: 49.5:1.0 v/v/v) was maintained at a flow rate of $1 \mathrm{~mL} / \mathrm{min}$. 


\subsection{Extraction and clean-up}

Ten milliliters volume of human urine was diluted with $10 \mathrm{~mL}$ of a solution of $5 \% \mathrm{NaHCO}_{3}$ in distilled water, gently mixed and filtered through Whatman No. 1 filter paper. Then, the filtered sample was clean-up through the OchraTest ${ }^{\mathrm{TM}}$ immunoaffinity column at a flow rate of about $1 \mathrm{drop} / \mathrm{s}$. The column was washed twice with $5 \mathrm{~mL}$ distilled water, and OTA was then slowly eluted from the column with $3 \mathrm{~mL}$ of methanol HPLC grade into a glass vial and column was dried by passing air through the column to collect the last drops of eluate. The eluted sample was evaporated to dryness under a gentle stream of nitrogen at ca. $50^{\circ} \mathrm{C}$ and the dry residue was dissolved in $150 \mu \mathrm{L}$ of the HPLC mobile phase. Finally, $50 \mu \mathrm{L}$ was injected onto HPLC column.

The IAC columns were regenerated according to Scott and Trucksess (1997). They were cleaned-up by passage with $5 \mathrm{~mL}$ of methanol followed by $20 \mathrm{~mL}$ of PBS solution and filled with PBS solution and stored until the next use. This regeneration was never made more than once.

\subsection{Sampling}

A total 60 samples of human urine were collected from healthy individuals living in Coimbra city (53 samples, $89.83 \%$ ) and the countryside in central part of Portugal (six samples, 10.17\%) in November 2004. All volunteers were asked to complete a rapid questionnaire about sex and age.

From 60 participants involved in this study, $43 \%$ $(n=26)$ were female and $57 \%(n=34)$ were male. They were aged between 19 and 82 years old $(46.2 \pm 17.8)$. The women were aged between 19 and 82 years $(43.8 \pm 19.2)$ and men were between 22 and 73 old $(47.9 \pm 16.1)$. All samples were stored at $4{ }^{\circ} \mathrm{C}$ until analysis.

\subsection{Chemical confirmation of OTA by methyl ester formation}

For confirmation, the OTA was converted into its methyl ester and two different procedures were evaluated.

In the first chemical confirmation performed according to the method of Zimmerli and Dick (1995) $200 \mu \mathrm{L}$ of the extract was diluted to $2.5 \mathrm{~mL}$ with methanol and $0.1 \mathrm{~mL}$ of $37 \%$ concentrated $\mathrm{HCl}$ was added. After standing overnight at room temperature, the methanol was evaporated and the residue dissolved in $250 \mu \mathrm{L}$ of mobile phase. OTA methyl ester was analyzed by liquid chromatography accordingly to that described above. A second chemical confirmation was carrying out following the procedure of Castegnaro et al. (1998). Sample extracts were evaporated to dryness, $150 \mu \mathrm{L}$ boron trifluoride $14 \%$ methanolic solution was added, and the mixture was left at $60^{\circ} \mathrm{C}$ for $10 \mathrm{~min}$. After evaporation, the residue was dissolved in $250 \mu \mathrm{L}$ of mobile phase. Finally $50 \mu \mathrm{L}$ was injected onto HPLC column.

\subsection{Assay with $\beta$-glucuronidase}

The assay with $\beta$-glucuronidase was performed according to the Orti et al. (1986) method. For hydrolysis, $5 \mathrm{~mL}$ of $\beta$-glucuronidase solution and $5 \mathrm{~mL}$ of $0.1 \mathrm{M} \mathrm{Na} \mathrm{Na}_{4}$ were added to $10 \mathrm{~mL}$ of urine, and the mixture was incubated overnight $(\sim 17 \mathrm{~h})$ at $37^{\circ} \mathrm{C}$. The hydrolyzed urine was extracted according to the procedure above and the extract was cleaned-up by the procedure described above.

\subsection{Statistical analysis}

Database management and statistical analysis were performed with the SPSS 10.0 Microsoft version. To test for significant differences between groups, the data were analyzed by ANOVA and then post-hoc analysis by Scheffe test was performed.

\section{Results and discussion}

\subsection{HPLC conditions optimization}

For the fluorescence detection a spectrum of OTA standard solution in HPLC mobile phase was obtained in order to optimize detection of OTA. The maximum emission wavelength at an excitation wavelength of $333 \mathrm{~nm}$, was found to be at $477 \mathrm{~nm}$.

The isocratic analysis under the conditions described allows the elution of OTA with good resolution (Fig. 1).

The mean retention time for OTA was $12.35 \mathrm{~min}$. On the basis of five parallel determinations, during five days, the precision standard deviation within-day and between-day (SD) of the OTA retention time, ranged from $0.02 \%$ to $0.04 \%$ and $0.03 \%$ to $0.07 \%$, respectively.

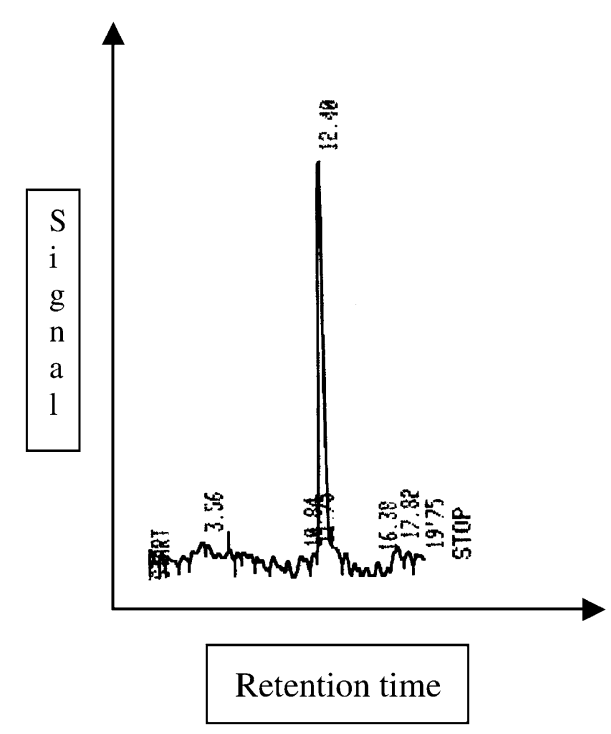

Fig. 1. Chromatogram of a standard solution of OTA at $10 \mathrm{ng} / \mathrm{mL}$. 


\subsection{Extraction and clean-up optimization}

In the literature, several methods are used to determine OTA from different type matrices, and in particular, the extraction step is a critical point of all methods, when the clean-up procedure employs IAC columns. The extraction method applied was based in Pascale and Visconti method (2001), with some modifications. After dilution with 5\% $\mathrm{NaHCO}_{3}$ and filtration the sample was cleaned up through IAC column. Ours results showed that $2 \mathrm{~mL}$ of methanol was not sufficient to elute all OTA. Therefore, to improve clean-up efficiency, after elution of OTA with $2 \mathrm{~mL}$ of methanol, a second elution with $1 \mathrm{~mL}$ of methanol was performed, and OTA peak was found in this second eluate. In next assay, OTA was eluted with $3 \mathrm{~mL}$ of methanol and after with next $1 \mathrm{~mL}$ of methanol. OTA was not found in this second eluate. So, our results indicate that $3 \mathrm{~mL}$ of methanol are necessary and sufficient to elute all OTA from IAC column. Since the eluted extracts were evaporated to dryness and reconstituted only in $150 \mu \mathrm{L}$ of HPLC mobile phase, a LOQ of $0.02 \mathrm{ng} / \mathrm{mL}$ of urine $(1.3 \mathrm{ng} / \mathrm{mL}$ of the extract injected) was achieved, with good clean blank chromatograms.

Thus, this method was applied to analyze OTA in the urine samples. The IAC columns were regenerated according to the Scott and Trucksess (1997) procedure. They were cleaned-up by passage with $10 \mathrm{~mL}$ of methanol followed by $25 \mathrm{~mL}$ of PBS, and stored filled with PBS solution at 2$8^{\circ} \mathrm{C}$. This recovery was never made more than once. The IAC columns control was made periodically and data obtained show good reproducibility, presenting a mean RSD of $7 \%$.

Although unsilanized glassware was not used, it was washed with an acidic solution in order to avoid loss of OTA from neutral solvents by salt formation, precipitation and/or adsorption onto glassware (Valenta, 1998; Entwisle et al., 2000). This procedure was just effective as inactivating glassware as silanization, and easier and not so costly. This contributes to a better accuracy and precision of the results.

\subsection{Analytical method validation}

In order to verify the absence of potential interfering substances around the retention time of OTA, a number of representative blank urine samples $(n=10)$ were analyzed in order to assess the specificity of the method. No interferences were observed in the region of interest where the OTA was eluted as is shown in the blank sample chromatogram (Fig. 2).

The calibration curves were obtained using the linear least squares regression procedure of the peak area versus the concentration, between 1 and $10 \mathrm{ng} / \mathrm{mL}, 1.0 ; 2.5 ; 5.0$ and $10.0 \mathrm{ng} / \mathrm{mL}$, respectively. The linearity for OTA, in the working standard solutions at three determinations of five concentration levels, was good as shown by the fact that the determination of the correlation coefficients $\left(r^{2}\right)$

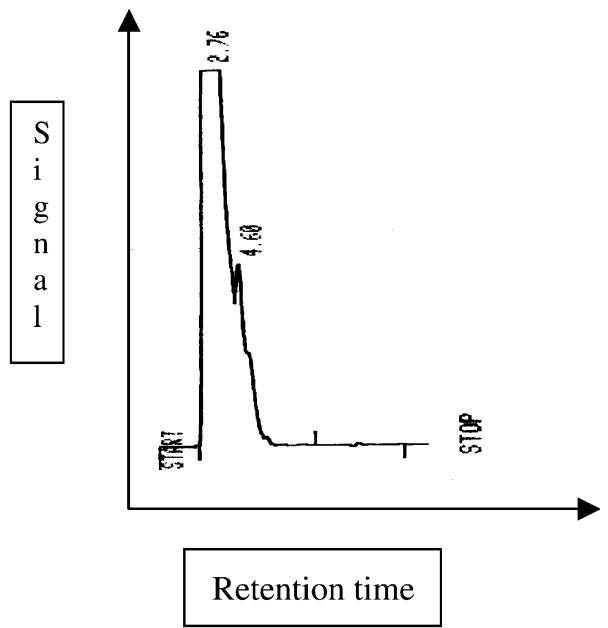

Fig. 2. Chromatogram of a blank sample.

are above 0.9990 for nine calibration curves, prepared in three different days.

The limit of quantification (LOQ) $0.02 \mathrm{ng} / \mathrm{mL}$ of OTA in urine, corresponding to $1.3 \mathrm{ng} / \mathrm{mL}$ of the extract injected, was determined by the signal-to-noise approach, defined as that level resulting in a signal-to-noise ratio of approximately 10:1.

Within-day accuracy and the precision data were determined by analyzing, on the same day, three replicates of spiked samples at three levels $(0.02,0.1$ and $0.5 \mathrm{ng} / \mathrm{mL})$ and one blank (to check interferences). The between-day accuracy and precision were also by extracting batches of three fortification levels and analyzing them on three different days. Accuracy and intra-day and inter-day precision data is shown in Table 1. Under our conditions, for the three fortification levels, recoveries from spiked urine samples for OTA reached very high values, greater than $90 \%$ and the relative standard deviation was less than 9\% demonstrating good method accuracy and precision.

\subsection{Confirmation by methylation of OTA}

The presence of OTA in selected positive sample was confirmed by formation of the OTA methyl ester and identified by comparing with the retention time of standard methyl ester of OTA.

Table 1

Accuracy and inter- and intra-assay validation results

\begin{tabular}{llll}
\hline $\begin{array}{l}\text { Fortification } \\
\text { level }(\mathrm{ng} / \mathrm{mL})\end{array}$ & $\begin{array}{l}\text { Recovery } \\
\text { mean }(\%)\end{array}$ & $\begin{array}{l}\text { RSD } \\
\text { within-day }\end{array}$ & $\begin{array}{l}\text { RSD } \\
\text { between-day }^{\mathrm{b}}(\%)\end{array}$ \\
\hline 0.02 & 90.6 & 5.4 & 8.9 \\
0.1 & 92.8 & 3.2 & 5.6 \\
0.5 & 96.0 & 2.9 & 4.3 \\
\hline
\end{tabular}

${ }^{\text {a }}$ Repeatability on the basis of three replicates at $0.02,0.1$ and $0.5 \mathrm{ng} / \mathrm{mL}$ within the same day.

b Three days repeatability on the basis of analysis at $0.02,0.1$ and $0.5 \mathrm{ng} /$ $\mathrm{mL} /$ day. 


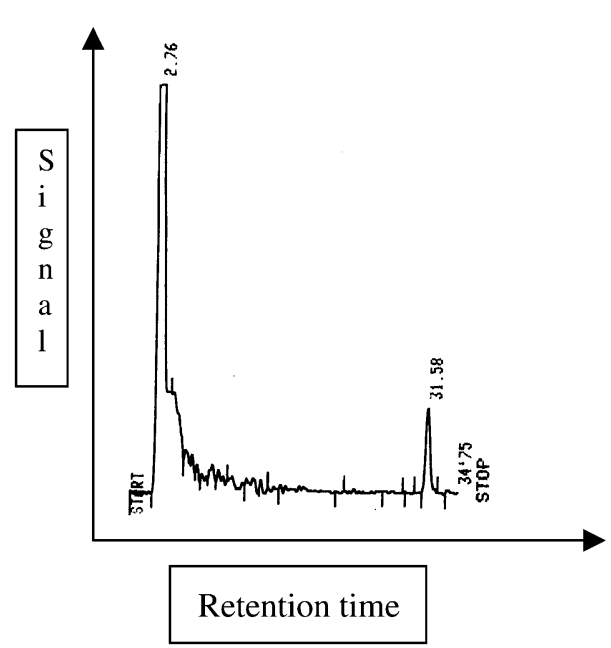

Fig. 3. Chromatogram of the OTA methyl ester.

Positive confirmation was based in disappearance of the OTA peak and appearance of new one, corresponding to OTA methyl ester, at retention time $31.58 \mathrm{~min}$ (Fig. 3). The use of boron trifluoride (Nesheim et al., 1992) and concentrated hydrochloric acid (Zimmerli and Dick, 1995) as catalysts to form the methyl ester of OTA were evaluated. A boron trifluoride in methanol solution (14\%) is used for the preparation of the methyl ester in most methods and is also part of the official AOAC method for OTA determination on corn and barley by HPLC (AOAC, 1997). For boron trifluoride-methanol reagent, the recovery rate of the methyl ester was higher $(93 \%)$ when compared with the method of Zimmerli and Dick (1995) (79\%) with good blanks.

Following the Nesheim et al. (1992) method the confirmation of OTA to methyl ester was performed in all samples containing levels upper than $0.02 \mathrm{ng} / \mathrm{mL}$ urine.

\subsection{Stability study}

Reference literature reports that OTA solutions in methanol are stable for several years at $-20^{\circ} \mathrm{C}$ (Valenta, 1998), but the stability of other OTA solutions prepared during the analytical process was assessed. OTA standard solutions in mobile phase were analyzed during $8 \mathrm{~h}$ under laboratorial conditions (temperature $\approx 22{ }^{\circ} \mathrm{C}$ ). The results obtained showed that OTA was stable under these conditions for this period of time.

Although, some authors did not find changes in OTA concentration in milk and pig kidney tissues, there are no data on the stability of OTA in naturally contaminated human biological material. Therefore, in this work a stability study in naturally contaminated urine samples was performed weekly during 1 month. Positive urine samples were prepared according the analytical procedure described above and inject into HPLC system. After one week we observed a decrease of $10 \%$ in the concentration of OTA, and after one month control the OTA concentration still maintain in this order value ( $10 \%$ lower).

\subsection{Study with $\beta$-glucuronidase}

Since a major portion of OTA is excreted in human urine, conjugated as glucuronides, and most of the analytical methods reported for OTA analysis in urine not include a previous hydrolysis, an assay with $\beta$-glucuronidase was performed in four naturally contaminated samples with $0.105,0.095,0.081$ and $0.092 \mathrm{ng} / \mathrm{mL}$ of OTA. After incubation with $\beta$-glucuronidase OTA was detected in the samples in higher amounts: $0.290,0.167,0.231$ and $0.195 \mathrm{ng} / \mathrm{mL}$, respectively. The hydrolysis procedure described here includes the addition of sodium sulphate to the urine sample to prevent interferences from the mucopolysaccharides that compete for the enzyme, according to the Orti et al. (1986) method. They included incubation with $\beta$-glucuronidase in their method for OTA determination in human urine, however, as naturally contaminated samples were not analyzed in this study, OTA conjugation with glucuronic acid in human urine could not be proven.

Based in ours first results we can assume that glucuronide conjugates were found in the human urine sample analyzed, but more assays should be undertaken in order to evaluate the extension of OTA conjugation with glucuronic acid in human urine.

\subsection{Evaluation of OTA in urine samples}

A total 60 samples of human urine were analyzed under the conditions described and OTA was confirmed in 42 of 60 urine samples $(70 \%)$, at concentrations above the LOQ, ranged between 0.02 and $0.105 \mathrm{ng} / \mathrm{mL}$ (Table 2).

A study reported urine levels for endemic nephropathy patients and for healthy individuals in Bulgaria (Maff, 1997). Urine from nephropathy patients contained OTA in the range $0.005-0.604 \mathrm{ng} / \mathrm{mL}$ healthy individuals from the same area had urinary levels between 0.005 and $0.043 \mathrm{ng} / \mathrm{mL}$, while OTA was not detected in the urine of individuals from a region that did not suffer from endemic nephropathy.

Table 2

Mean, minimum and maximum levels ( $\mathrm{ng} / \mathrm{mL}$ ) and frequency (\%) of OTA in urine samples

\begin{tabular}{llllc}
\hline & Mean & Min & Max & $\%$ Frequency \\
\hline Total & 0.038 & 0.021 & 0.105 & 70 \\
Male & 0.039 & 0.022 & 0.105 & 79 \\
$20-39$ & 0.029 & 0.022 & 0.040 & 91 \\
$40-60$ & 0.062 & 0.036 & 0.105 & 87 \\
$61-70$ & 0.034 & 0.025 & 0.050 & 25 \\
$71-80$ & 0.032 & 0.028 & 0.050 & 75 \\
Female & 0.037 & 0.021 & 0.070 & 58 \\
$<20$ & 0.023 & 0.023 & 0.023 & 100 \\
$20-39$ & 0.038 & 0.030 & 0.050 & 40 \\
$40-60$ & 0.031 & 0.021 & 0.050 & 60 \\
$61-70$ & 0.060 & 0.060 & 0.060 & 67 \\
$71-82$ & 0.036 & 0.021 & 0.070 & 100 \\
\hline
\end{tabular}


In other study, the urine of individuals, living in BENendemic villages OTA was found more often than in those in non-endemic villages, and the highest levels were seen in patients with BEN or urinary tract tumors (PetkovaBocharova et al., 2003).

To test for statistically significant differences between groups, the data were analyzed by ANOVA and then post-hoc analysis by Scheffe test was performed. Significant differences were only observed between gender to the group aged between 20 and 39 years $(P=0.044)$.

\section{Conclusions}

This preliminary study has shown that OTA is widespread in the sample population.

We can assume that glucuronide conjugates were found in positive human urine samples analyzed. So, in analytical methodology applied to urine samples, a previous hydrolysis should be included, in order to determine free OTA and OTA glucuronides.

The collection of urine is less invasive than blood for follow up of population exposure, and analysis of OTA in urine is a good marker and very useful route to monitor OTA exposure of populations. The finding of that low concentration of OTA in human urine samples, stresses the problem of their toxicological significance. Given the lack of OTA levels in human urine, the conducting of these studies in order to evaluate food safety is highly recommended. Further work is currently underway to monitor Portuguese population as a whole.

\section{Acknowledgements}

We gratefully thank FCT and POCTI (FEDER) for financial support of this study.

\section{References}

Álvarez, L., Gil, G., Ezpeleta, O., Garcia-Jalón, J., de Cerain, L., 2004. Immunotoxic effects of OTA in wistar rats after oral administration. Food Chem. Toxicol. 42, 825-834.

AOAC International, 1997. Official Methods of Analysis of AOAC International, 16th ed., 3rd revision. AOAC International, Gaithesburg.

Biffi, R., Munari, M., Dioguardi, L., Ballabio, C., Cattaneo, A., Galli, C.L., Restani, P., 2004. Ochratoxin A in conventional and organic cereal derivatives: a survey of the Italian market, 2001-02. Food Addit. Contam. 21, 586-591.

Blesa, J., Berrada, H., Soriano, J.M., Moltó, J.C., Mañes, J., 2004. Rapid determination of ochratoxin $\mathrm{A}$ in cereals and cereal products by liquid chromatography. J. Chromatogr. A 1046, 127-131.

Castegnaro, M., Mohr, U., Pfohl-Leszkowicz, A., Estéve, J., Steinmann, J., Tillmann, T., Michelon, J., Bartsch, H., 1998. Sex- and strainspecific induction of renal tumors by ochratoxin $\mathrm{A}$ in rats correlates with DNA adduction. Int. J. Cancer 77, 70-75.

Dinis, A.M., Lino, C.M., Baeta, M.L., Pena, A., Silveira, M.I.N., 2003a. Levels of ochratoxin A in healthy people and nephropathy patients in central zone of Portugal. Rev. Toxicol., 119.

Dinis, A.M., Lino, C.M., Baeta, M.L., Pena, A., Silveira, M.I.N., 2003b. Levels of ochratoxin A in serum from urban and rural populations of central zone of Portugal. Rev. Port. Farm. LII, 187.
Entwisle, A.C., Williams, A.C., Mann, P.J., Slack, P.T., Gilbert, J., 2000. Liquid chromatographic method with immunoaffinity column cleanup for determination of ochratoxin A in barley: collaborative study. J. AOAC Int. 83, 1377-1383.

Gekle, M., Sauvant, C., Schwerdt, G., 2005. Ochratoxin A at nanomolar concentrations: A signal modular in renal cells. Mol. Nutr. Food Res. 49, 118-130.

IARC Monograph on the Evaluation of Carcinogenic Risks to Humans, 1993. IARC, Lyon, France, p. 489.

Li, S., Marquardt, R.R., Frohlich, A.A., Vitti, T.G., Crow, G., 1997. Pharmacokinetics of ochratoxin A and its metabolites in rats. Toxicol. Appl. Pharmacol. 145, 82-90.

Maff, UK, 1997. Food surveillance information sheets survey of aflatoxins and ochratoxin A in cereals and retail products, No. 130 [on line]. [consult. 20/10/2004]. Available on: <http://www.maff.gov.uk/food/ infsheet/1999/no185/185ochra.htm $>$.

Monaci, L., Tantillo, G., Palmisano, F., 2004. Determination of ochratoxin $\mathrm{A}$ in pig tissues by liquid-liquid extraction and clean-up and high-performance liquid chromatography. Anal. Bioanal. Chem. 378, 1777-1782.

Nesheim, S., Stack, M.E., Trucksess, M.W., Eppley, R.M., Krogh, P., 1992. Rapid solvent-efficient method for liquid chromatographic determination of ochratoxin A in corn, barley, and kidney: collaborative study. J. AOAC Int. 75, 481-487.

Orti, D.L., Hill, R.H., Liddle, J.A., Needham, L.L., 1986. High performance liquid chromatography of mycotoxin metabolites in human urine. J. Anal. Toxicol. 10, 41-45.

Pascale, M., Visconti, A., 2001. Rapid method for the determination of ochratoxin A in urine by immunoaffinity column clean-up and high-performance liquid chromatography. Mycopathologia 152, 9195.

Pena, A., Lino Cerejo F., C.M., Silveira, M.I., 2005. Determination of ochratoxin $\mathrm{A}$ in portuguese rice samples by high performance liquid chromatography with fluorescence detection. Anal. Bioanal. Chem. 382, 1288-1293.

Petkova-Bocharova, T., Castegnaro, M., Pfohl-Leszkowitz, A., Garren, L., Grosso, F., Nikolov, I., Vrabcheva, T., Dragaci, S., Chernozemsky, I.N., 2003. Analysis of ochratoxin A in serum and urine of inhabitants from an area with Balkan Endemic Nephropathy: a one month follow up study. Facta Univ. 10, 62-68.

Pfohl-Leszkowicz, A., Petkova-bocharova, T., Chernozemsky, I.N., Castegnaro, M., 2002. Balkan endemic nephropathy and associated urinary tract tumours: a review on aetiological causes and the potential role of mycotoxins. Food Addit. Contam. 19, 282-302.

Scott, M., Trucksess, W., 1997. Application of immunoaffinity columns to mycotoxin analysis. J. AOAC Int. 80, 941-949.

Studer-Rohr, I., Schlatter, J., Dietrich, D.R., 2000. Kinetic parameters and intraindividual fluctuations of ochratoxin A plasma levels in humans. Arch. Toxicol. 74, 499-510.

Thuvander, A., Paulsen, J.E., Axberg, K., Johansson, N., Vidnes, A., Enghardt-Barbieri, H., Trygg, K., Lund-Larsen, K., Jahrl, S., Widenfalk, A., Bosnes, V., Alexander, J., Hult, K., Olsen, M., 2001. Levels of ochratoxin A in blood from Norwegian and Swedish blood donors and their possible correlation with food consumption. Food Chem. Toxicol. 39, 1145-1151.

Valenta, H., 1998. Chromatographic methods for the determination of ochratoxin A in animal and human tissues and fluids. J. Chromatogr. A $815,75-92$.

Visconti, A., Pascale, M., Centonze, G., 2000. Determination of ochratoxin $\mathrm{A}$ in domestic and imported beers in Italy by immunoaffinity clean-up and liquid chromatography. J. Chromatogr. A 888, 321326.

Zimmerli, B., Dick, R., 1995. Determination of ochratoxin A at the ppt level in human blood, serum, milk and some foodstuffs by highperformance liquid chromatography with enhanced fluorescence detection and immunoaffinity column clean-up: methodology and Swiss data. J. Chromatogr. B 666, 85-99. 